\title{
2006-2050: ETHICS, SOCIAL RESPONSIBILITY, AND GLOBAL AWARENESS IN THE ENGINEERING TECHNOLOGY CURRICULUM
}

\section{S. David Dvorak, University of Maine-Orono}

David Dvorak is Professor of Mechanical Engineering Technology and Director of the School of Engineering Technology at the University of Maine. He joined the UMaine faculty in 1988. From 1982 to 1988 he worked at GE aircraft engines in Cincinnati, Ohio. Dr. Dvorak received B.S. and M.S. degrees in Mechanical Engineering from the University of Illinois at Urbana-Champaign in 1981 and 1982 respectively, and a Ph.D. in Physics from the University of Maine in 1998. He is a Registered Professional Engineer in Maine and Ohio. Dr. Dvorak is active in ASME and ASEE, recently serving on the Executive Board of the Engineering Technology Leadership Institute (ETLI).

\section{Ronald Fulle, Rochester Institute of Technology}

Assistant Professor Ron Fulle has been teaching at RIT since 1998 in the Telecommunications Engineering Technology Program. He received his MS in Telecommunications from the U of Colorado-Boulder and BS in Mathematics from SUNY Oswego. He has over 25 years of telecom industry experience with a large US based telecommunications company. 


\title{
Ethics, Social Responsibility, and Global Awareness in the Engineering Technology Curriculum
}

\begin{abstract}
As globalization affects the technology-based companies and industries that form our constituents, it becomes increasingly important that our students become aware of these issues within the context of their engineering technology curriculum. In addition, TAC/ABET specifies educational outcomes in these areas that must be assessed and demonstrated by our students prior to graduation. For these reasons, it is necessary and appropriate that we imbed instruction in ethics, social responsibility, professionalism, and global awareness into the fabric of our undergraduate culture. This paper describes a workshop given at the 2005 Engineering Technology Leadership Institute, held in Rochester New York on October 1-3. The workshop involved 16 faculty and administrators from two-year and four-year programs at 13 different institutions. In addition to describing the workshop for those who were not able to attend, the paper draws conclusions and points out a "best practice" approach for future workshops.
\end{abstract}

\section{INTRODUCTION}

The importance of ethics, social responsibility, and global awareness in the practice of engineering cannot be overstated. This paper will focus on ethics; it is also proposed that studying ethics within the broader context of social responsibility and global awareness enhances the students learning experience and may bring a more valuable engineer and citizen to society. In fact, Globig ${ }^{1}$ discusses the importance of defining engineering ethics within a broader "global ethic" based on universal moral ideals that transcend culture-specific mores.

Almost all engineering and engineering technology programs incorporate ethics education at some level, either as a separate course or distributed within other courses. The approach used in the workshop described in this paper focuses on what engineering technology educators can do to enhance the ethical education of their students by incorporating content and learning activities within the so-called technical portion of the curriculum.

\section{Engineering Technology Leadership Institute}

The Engineering Technology Leadership Institute (ETLI) is the leadership development activity of the Engineering Technology Council of ASEE. The purpose of the ETLI is to bring together engineering technology leaders and educators from two-year and four-year institutions and discuss issues of common interest and concern ${ }^{2}$. The stated goals of ETLI are

- To prepare leadership personnel through faculty and administrative development;

- To enable participants to broaden their knowledge of engineering technology leadership issues;

- To discuss problems of management in an engineering technology environment;

- To provide specific leadership training;

- To provide an opportunity to exchange ideas with fellow educators;

- To provide participants with an opportunity to share their successes in leadership areas; 
- To provide an opportunity to exchange ideas with industrial leaders.

The ETLI is a two or three-day event hosted by an engineering technology institution in the fall. In October 2005, the authors presented a workshop regarding ethics education. The purpose of the workshop was to provide background and motivation for the integration of ethics into the Engineering Technology curriculum and, to that end, involve the participants in exercises (case studies) of varying complexity.

\section{ETLI Ethics Workshop: BaCkground Presentation}

In order to build a small but common base from which to work, the background for a limited basis of ethical philosophies and decision making was presented. Participants were then able to approach the case studies within the context of this brief overview.

\section{Review of the Relevant TAC/ABET criteria}

The TC2K Criterion 2 states the educational outcomes of any engineering technology program ${ }^{3}$. These outcomes are statements of student performance at the time of graduation. There are eleven separate outcomes (often listed a through $\mathrm{k}$ ). Several of the outcomes focus on technical competence, but there are a number that address broader issues which, not surprisingly, many educators find challenging to address:

e. an ability to function effectively on teams,

g. an ability to communicate effectively,

h. a recognition of the need for, and an ability to engage in lifelong learning,

i. an ability to understand professional, ethical and social responsibilities,

j. a respect for diversity and a knowledge of contemporary professional, societal and global issues, and

k. a commitment to quality, timeliness, and continuous improvement.

Ethics instruction addressed in the 2005 ETLI Ethics Workshop clearly supports outcome (i) above, while studying ethics in the context of global issues also supports outcome (j). In addition, if student-centered learning activities incorporating the principles of active and reflective learning are used, outcome (e) and (g) are also supported. Finally, open-ended exercises involving published engineering Codes of Conduct support outcome (h).

\section{Theories of Ethics Presented in the Workshop}

How do we define ethics and approach the study of ethics? Ethics is the study of what constitutes right and wrong and/or good and bad conduct. Engineering ethics is a branch of practical ethics. It is the study of what constitutes right and wrong (or good and bad) in an engineering context. There are many theories of ethics. Virtue ethics is usually attributable to Aristotle. Before Aristotle, Plato and others taught that one must "know the good." However, Aristotle taught that it was not enough to just know the good, one must "do the good." Aristotle made that leap and taught that an ethical person must put "the good" into practice by doing the good. Virtue ethics also introduced the idea of a "midpoint virtue," where moderation is the goal, 
and extremes in thought and action (vices) are to be avoided. Experience has shown that many engineering technology students remember this distinction if we take a bit of story license and introduce Aristotle as "the first Engineering Technologist."

Consequentialist and non-Consequentialist theories further expanded aspects of the philosophy of ethics. Consequentalist philosophers hold that the rightness of an action is determined exclusively by the outcomes of the action. Non-consequentalists believe that the consequences of an action are morally significant, but additional factors are also important in determining the most moral course of action.

Theories of justice address the broader consideration of social structure, such as individual rights, fairness, and equality. Harvard University's John Rawls' post WWII philosophy proposed the interesting if highly ideal social contract theory of ethics ${ }^{4}$. Rawls envisions a society where certain fundamental liberties are guaranteed to each person, and the (inevitably unequal) distribution of natural talent is viewed as a common asset for the benefit of all society members, including the least fortunate.

Deontological theory or as students sometimes call it "duty theory" is a philosophy that is easily understood by students. They seem to enjoy Ross ${ }^{4}$ in which ethical thought is framed into seven "duties" as listed and described below:

1 Duties of Fidelity: keep promises both explicit \& implicit;

2 Duties of Reparation: pay compensation for harm/injuries caused;

3 Duties of Gratitude: return Favors;

4 Duties of Justice: how should we distribute goods, services (merit, need, effort, other);

5 Duties of Beneficence: try to help others;

6 Self-improvement: enhance our virtue \& intelligence;

7 Duties of Nonmalfeasance: don't harm others.

Ethics has many different, varying and sometimes conflicting philosophies or viewpoints. However, at its essence, there is always the issue of right and wrong, good or bad and some basic moral principles. It is ideal if one can solve ethical problems by knowing the good and acting on that knowledge. In these cases, this "good acting and doing" yields no violation of moral principles. However, very often, an ethical problem may reduce itself to a choice between two competing pathways in which, regardless which path is chosen, there would be a transgression or harm of a moral imperative due to the path not taken. This would be an ethical dilemma because each choice would violate at least one moral principle. Much can be learned by attempting to solve these ethical dilemmas.

Ethics can also be thought of in a more restrictive sense as permissible standards of conduct that govern members of a particular group. This interpretation actually allows the development of specific and detailed codes that vary depending on the group. Most organized professions have codified ethical behavior as applied to their particular area of expertise and practice.

Engineering society codes of ethics fall into this type of ethical framework.

In summary, three aspects of ethics ${ }^{5}$ were presented in the workshop:

1. Ethics as a moral theory (philosophy), 
2. Ethics as ordinary morality (duties or moral principles as described above),

3. Ethics as morally permissible standards of conduct that govern members of a group (professional ethics).

\section{Ethical Decision Making}

Ethical decision making can be viewed as a special case of decision making ${ }^{6}$. For engineering students, decision making can be demystified if framed in terms of problem solving methodologies. The first step in any problem-solving method is to make sure that the problem is carefully defined. This is true in the solving of technical problems, and it is no less true with ethical problems. The initial step, then, is to determine the facts of the case, carefully separating emotional response from what is factual, what is said from what is known, and what is known from what is inferred. Once the facts of the case are well defined then one must determine what moral principles are being violated and whether or not there is a moral dilemma. Tracking and determining the harms and determining what to do based upon one's goals brings this problem solving practice to a conclusion. An example of a process used for ethical problem solving is given in Appendix A.

In summary, the instructional model used in the ETLI Ethics Workshop included a basic background presentation on the theory and definition of basic moral principles and the introduction of a simple problem-solving method. This background presentation was followed by active learning exercises involving case studies, as described in the next section.

\section{ETLI ETHICS WORKSHOP: EXERCISES}

The workshop was designed to be experiential in nature. The focus of the workshop was to have the participants experience the case studies with the same "learner-centered" discussion and decision-making process that an engineering technology student might experience within their programs of study.

Once a limited set of ethical philosophies was introduced, workshop participants were presented with a case study involving a workplace situation that was not engineering-related. The participants were asked to work on the case study using the one-page guide for ethical decisionmaking (appendix A). This guide outlines a process for ethical problem solving that is applicable to a wide range of ethical situations. The workshop participants then discussed the case in small groups, and after about 20 minutes the workshop reassembled and each group reported their findings. These findings included the process they used to resolve the ethical situation, starting with the facts of the case, determining the ethical principles involved, and a recommendation for action to resolve the situation. The workshop presenters then reviewed codes of ethics from two different engineering societies (ASME and IEEE) and discussed similarities and differences. Each group was then given an engineering oriented case, based on NSPE Board of Ethical Review cases ${ }^{7}$. Each group was given a different case. After 30 minutes of discussion, each group presented to the other groups the background of their case, and the process used to address the ethical situation. These cases typically involved moral dilemmas, and were considerably more challenging, being also connected with broader society and cultural issues.

\section{DISCUSSION}


The workshop participants appreciated that several of the case studies went beyond practices described in the codes of ethics, and touched on more fundamental moral principles. There was considerable discussion on whether or not "professional ethics" could be separated from "personal ethics." Some participants felt that there was some overlap between personal and professional ethics, and perhaps one should strive for complete congruency, where personal and professional ethics are the same. As James Globig has observed, "It is not logical to conduct our professional life on a moral basis different from our personal life." This in an important realization, since professional codes of ethics govern behavior in a restricted working environment, and are of limited applicability when broader issues are involved, as when engineers on interdisciplinary teams, or especially when complex situations require a global awareness involving multiple cultures.

The optimal delivery of ethics in the curriculum was also an important point of discussion: Is it better to teach ethics in engineering technology within a special class or as a subject intertwined within the core curriculum? At Rochester Institute of Technology, the engineering technology programs have a separate class in ethics and project management ${ }^{8}$, while at other institutions attempts are being made to include ethics throughout the curriculum. Faculty familiarity and expertise is necessary regardless of approach however, less quantity faculty with such expertise is necessary when ethics is within a special course. Also, if ethics is taught across the curriculum, often it may be optimum to have a required initial course that helps provide the basics of ethics----thereby allowing the core courses to concentrate on applications within the technology being taught. Generally, it was the consensus that providing ethics across the curriculum might be a good target end goal.

\section{CONCLUSIONS}

The goals of this workshop were:

$>$ To familiarize engineering technology leaders and educators with the basics of ethical problem solving.

$>$ To have participants actually experience an instructional model for incorporating an ethics into the engineering technology curriculum.

\section{Future Work}

The two goals given above were certainly met. However, had we clearly stated a third goal--that of having participants return to their home schools, programs and courses and better incorporate ethics and ethical decision making into their engineering technology curricula, the participants would have been better served. One suggestion for future workshops would be to include a presentation and discussion of available resources for faculty. Another suggestion (based on a reviewer comment) would be to have workshop participants commit to completing in a survey 6 months after the workshop which would measure how well they put these concepts into practice. Remember, it is not enough to "know the good," one must "do the good!"

\section{REFERENCES}


1. Globig, James E., "Applying a Global Ethic in Engineering Organizations," Proceedings of the American Society for Engineering Education Annual Conference \& Exposition, 2001.

2. ETLI website: www.sinclair.edu/academics/egr/pub/etli/

3. ABET website: www.abet.org

4. Shaw, William H., and Barry, Vincent, Moral Issues in Business, $9^{\text {th }}$ ed., Thomson \& Wadsworth, 2003.

5. Davis, Michael, "Ethics Across the Curriculum: the Second Decade," Center for the Study of Ethics in the Professions, Illinois Institute of Technology, 1998.

6. Shuman, Larry J., Sindelar, Mark F. , Besterfield-Sacre, Mary, Wolfe, Harvey, Pinkus, Rosa L., Miller, Ronald L., Olds, Barbara M., and Mitcham, Carl, "Can Our Students Recognize and Resolve Ethical Dilemmas?" Proceedings of the American Society for Engineering Education Annual Conference \& Exposition, 2004.

$7 \quad$ NSPE Board of Ethical Review: www.niee.org/cases/

8. Fulle, Ron, Richardson, Carol, and Zion, George, "Building Ethics and Project Management into Engineering Technology Programs," Proceedings of the American Society for Engineering Education Annual Conference \& Exposition, 2004. 


\section{APPENDiX A:}

\section{A Brief Guide to Ethical Decision-making}

$0 . \quad$ What are the facts of the case?

a. Be objective and distinguish.

i. Your emotional response from what is true

ii. What is said from what is known.

iii. What is known directly from what is inferred.

b. Discovering the facts is a continuing process: facts thought to be irrelevant may be seen later to be relevant.

1. Keep in mind the kinds of moral problems there can be:

a. Violation of such moral principles as e.g.

i. Principle of Autonomy: A person has the right to decide for what to do or omit from doing regarding those matters that affect him or her. Such decisions are (best) made voluntarily when fully informed and competent.

ii. Principle of Nonmaleficence: One ought not to harm others.

iii. Principle of Beneficence: One ought to help others, either by mitigating harmful conditions or by providing benefits.

iv. Principles of Justice:

1. One ought to treat like cases alike, and unlike cases unalike.

2. Whatever the basis for distributing benefits and burdens, it should not include such morally irrelevant contingencies as sex, race, color, age, income, social position, etc.

b. Moral dilemmas: one has a moral dilemma when one has incompatible choices, each supported by (at least) one moral principle, so that no matter what one does, one will have violated one moral principle.

2. Getting a handle on the problem:

a. Tracking harms - One helpful way of entry into a complex moral problem is to track the harms that have occurred and are likely to occur without intervention. It is useful, in this regard, to ask....

i. Who is affected

ii. What rights, of which people, have been harmed so far?

iii. Which interests, of which people, have been harmed so far?

b. Determining goals - One helpful way of proceeding after tracking harms is to determine what one's goals are in the case in question, both long-term and short-term.

3. Deciding what to do

a. Be sure a real moral problem exists. Disagreements often occur which, for all their emotion, are not really moral problems because no conflict of interest or rights exists.

b. If the problem is the violation of a moral principle - for no good moral reason (that is, no other moral principle justifies what is causing the problem), then no moral problem exists: violating a moral principle is just wrong.

c. If a moral dilemma exists, then much depends upon the details of the case and how one ranks the principles involved.

i. Tracking various solutions - One ought to figure out the long-term effects of possible solutions to the problem

ii. Applying principles -- One ought to apply at least the following principles in descending order:

1. Do not deny anyone's rights. So if a right conflicts with an interest, the right wins.

2. Minimize the harm consistent with recognizing the rights of those involved.

3. Maximize the harm consistent with recognizing the rights of those involved

d. One short way to test one's solution is to ask, 'Would I agree to be treated in the way I am treating those involved?' But the proper answer presupposes that one is objective and unbiased. 